\title{
Measurement of Aldehyde Dehydrogenase 1 Expression Defines a Group with Better Prognosis in Patients with Non-Small Cell Lung Cancer
}

\author{
Anastasios Dimou, ${ }^{*}$ Veronique Neumeister, ${ }^{*}$ \\ Seema Agarwal, ${ }^{*}$ Valsamo Anagnostou, ${ }^{\dagger}$ \\ Konstantinos Syrigos, ${ }^{\ddagger}$ and David L. Rimm* \\ From the Departments of Patbology* and Internal Medicine ${ }^{\dagger}$ and \\ the Section of Medical Oncology, ${ }^{\ddagger}$ Department of Internal \\ Medicine, Yale Cancer Center, Yale University School of \\ Medicine, New Haven, Connecticut
}

Aldehyde dehydrogenase 1 (ALDH1) has been suggested as a surrogate biomarker for cancer stem cells in breast cancer and other tumors. We quantitatively measured ALDH1 in two large cohorts of patients with non-small cell lung cancer (NSCLC) and investigated its prognostic value. The AQUA method of quantitative immunofluorescence was used to measure ALDH1 in 134 patients with NSCLC from Yale University and 296 patients with NSCLC from Sotiria and Patras University hospitals in Greece, using tissue microarrays. Patients were classified as positive or negative for ALDH1 based on the detection threshold for quantitative immunofluorescence. Patients with squamous cell carcinoma had higher scores than patients with adenocarcinoma. Detectable ALDH1 predicted better prognosis in both cohorts $(P=\mathbf{0 . 0 0 3 5}$ for the Yale cohort; $P=0.0238$ for the Sotiria/Patras cohorts). The effect of ALDH1 expression was independent of clinicopathologic factors in the Yale cohort (risk ratio $=3.2, P=0.0008$ ), but did not reach significance in the Sotiria/Patras cohort (hazard ratio $=$ 1.51, $P=0.08$ ). Among patients with adenocarcinoma, the ALDH1-negative group had shorter survival compared with the ALDH1-positive group in the Yale cohort $(P=0.00001)$, but not in the Sotiria/Patras cohort $(P=0.45)$. Unlike breast cancer, in which ALDH1 expression predicts poor outcome, in NSCLC our exploratory and retrospective study indicates that ALDH1 expression is associated with favorable outcome. (Am J Pathol 2012, 181:1436-1442; http://dx.doi. org/10.1016/j.ajpath.2012.06.037)
Non-small cell lung cancer (NSCLC) is the leading cause of cancer-related death worldwide. ${ }^{1}$ To date, tumor stage is the most informative baseline information that defines prognosis and aids treatment decisions. The prognostic potential of histology type, age, sex, and other traditional patient characteristics has been recognized in several reports, but is limited. ${ }^{2-6}$ On the other hand, an increasing body of literature indicates molecular biomarkers as determinants of prognosis, thus pointing to the importance and feasibility of classifying patients with NSCLC according to unique molecular profiles.

Aldehyde dehydrogenase (ALDH) belongs to a family of detoxifying enzymes that convert aldehydes to their corresponding carboxylic acids. ${ }^{7,8}$ Members of this family are present in many types of normal tissues. ALDH1 has been suggested as a surrogate biomarker for hematopoietic stem cells. ${ }^{9}$ In addition, ALDH1 is a variant reported to be enriched in cells with stem cell properties in several types of malignancies. ${ }^{10}$ In this context, ALDH1-positive cells demonstrate asymmetrical differentiation and tumor-initiating capacity in breast cancers, ${ }^{11}$ and ALDH1 positivity predicts worse prognosis and resistance to chemotherapy in those tumors. ${ }^{12}$ Nevertheless, it is controversial whether a single marker can serve as an adequate surrogate for cancer stem cell detection. ${ }^{13}$ There is evidence to suggest that the combination

Supported in part by the Oncology Unit of the Sotiria General Hospital, Athens, Greece, and Department of Public Health Grant \#2009-0097 from the State of Connecticut (D.L.R.).

Accepted for publication June 20, 2012.

D.L.R. declares a duality of interest as a cofounder, consultant, and stockholder of HistoRx. This company was founded by Yale University and is the exclusive Yale licensee of the AQUA technology developed in the laboratory of D.L.R. AQUA is a registered trademark of HistoRx.

Supplemental material for this article can be found at http://ajp. amjpathol.org or at http://dx.doi.org/10.1016/j.ajpath.2012.06.037.

Current address A.D.: Department of Internal Medicine, Albert Einstein Medical Center, Philadelphia, Pennsylvania.

Address reprint requests to David L. Rimm, M.D., Ph.D., Department of Pathology, BML 116, Yale University School of Medicine, 310 Cedar St., PO Box 208023, New Haven, CT 06520-8023. E-mail: david.rimm@ yale.edu. 
Table 1. Clinicodemographic Data for Two Cohorts of NSCLC Patients, with AQUA Score for ALDH1 Expression

\begin{tabular}{|c|c|c|c|c|}
\hline \multirow[b]{2}{*}{ Variable } & \multicolumn{2}{|c|}{ Yale cohort } & \multicolumn{2}{|c|}{ Sotiria/Patras cohort } \\
\hline & No. (\%) & AQUA score & No. (\%) & AQUA score \\
\hline All patients & $134(100)$ & 7039 (619-24,090) & $296(100)$ & $5429(606-23,424)$ \\
\hline \multicolumn{5}{|l|}{ Age at diagnosis (years) } \\
\hline$\leq 65$ & $63(47)$ & $6385(787-23,738)$ & $136(45)$ & $5429(670-23,424)$ \\
\hline$>65$ & $71(53)$ & $7434(619-24,090)$ & $138(46)$ & $4948(606-22,662)$ \\
\hline Missing data & 0 & & $22(9)$ & \\
\hline \multicolumn{5}{|l|}{ Sex } \\
\hline Male & $70(52)$ & 8102 (619-24,090) & $242(82)$ & $5660(670-23,424)$ \\
\hline Female & $64(48)$ & $6448(787-21,989)$ & $35(12)$ & $12,853(606-22,662)$ \\
\hline Missing data & 0 & & $19(6)$ & \\
\hline \multicolumn{5}{|l|}{ Histotype } \\
\hline$A C$ & $83(61)$ & 5077 (619-21,989) & $111(37.5)$ & 2703 (670-21,133) \\
\hline LCC & $7(5)$ & 7696 (978-24,090) & $2(0.6)$ & 899-1358 \\
\hline SCC & $26(20)$ & $13,232(1071-23,738)$ & $146(49.4)$ & $9139(606-23,424)$ \\
\hline Other/mixed & $18(13)$ & $8035(832-21,936)$ & $17(5.7)$ & $3922(935-20,502)$ \\
\hline Missing data & $0(0)$ & & $20(6.8)$ & \\
\hline \multicolumn{5}{|l|}{ Stage } \\
\hline $\mid \vec{A}$ & $47(35)$ & $9977(619(24,090)$ & $23(8)$ & $5202(938-21,360)$ \\
\hline IB & $21(15)$ & $3745(846-19,278)$ & 66 (22) & $5971(606-22,226)$ \\
\hline II & $18(13)$ & $5940(656-21,989)$ & $79(26)$ & $7190(674-23,424)$ \\
\hline III & $33(24)$ & 6316 (787-21,936) & $78(27)$ & $4006(674-22,662)$ \\
\hline IV & $12(9)$ & $3076(809-17,791)$ & $29(27)$ & $2783(670-21,634)$ \\
\hline Missing data & $3(2)$ & & $21(7)$ & \\
\hline
\end{tabular}

AQUA scores were calculated as the ratio of the sum of the target pixels in the tumor mask divided by the area of the tumor mask and are expressed as median (range).

AC, adenocarcinoma; Cl, confidence interval; HR, hazards ratio; LCC, large cell carcinoma; NSCLC, non-small cell lung cancer; SCC, squamous cell carcinoma.

of ALDH1 and CD44 defines the population of cancerinitiating cells more adequately than either protein alone. ${ }^{14,15}$ Furthermore, different isoforms of ALDH might be important in stem cell biology. ${ }^{16}$

Typically, ALDH1 is assessed with a flow cytometrybased system. ${ }^{17}$ Immunohistochemistry is another popular method for in situ detection at the protein level. IHC in general is prone to certain limitations related to suboptimal standardization and validation assays, as well as subjectivity in the interpretation of the results. ${ }^{18}$ We assessed the expression of ALDH1 in two large cohorts of patients with NSCLC, using an immunofluorescencebased, objective, and quantitative approach.

\section{Materials and Methods}

\section{Patient Cohorts}

The first cohort of NSCLC cases $(n=134)$ was accrued by serial collection of formalin-fixed, paraffin-embedded tissue obtained from the Department of Pathology at Yale University (New Haven, CT). The second cohort $(n=296)$ was obtained from the pathology departments of Sotiria General Hospital (Athens, Greece) and Patras University Hospital (Rion, Greece) in a similar manner. In the Yale cohort, patients were diagnosed during the period 1993 to 2003, with all but three patients diagnosed after 1995 . The median age was 66 years (range, 42 to 86 years), and median follow-up time was 27.1 months (range, 0.23 to 133 months). In the Sotiria/Patras cohort, patients were diagnosed during the period 1990 to 2008, with all but one patient diagnosed after 1999. The median age was 65 years (range, 34 to 84 years), and median follow-up time was 20 months (range, 0.1 to 223). Histological diagnosis was based on the World Health Organization classification for lung tumors, ${ }^{19}$ and stage was determined using the American Joint Committee for Cancer staging system. ${ }^{20}$ Patient demographics for the two cohorts are given in Table 1. The study was approved by the institutional review boards of all centers. Written informed consent was obtained for each case before inclusion in the study.

\section{Tissue Microarray Construction}

Formalin-fixed, paraffin-embedded tissue blocks were scored twice in representative tumor areas using 0.6-mm cores and were arrayed into a recipient block at the Yale Pathology Tissue Services facility. Formalin-fixed, paraffinembedded pellets were prepared using standard cell block methods from the cell lines MCF7, H1299, H1355, H1666, HCC193, HCC2279, HT29, H2126, A549, SW480, HCC15, $\mathrm{H} 1819, \mathrm{~A} 431$, and $\mathrm{H} 1650$ were purchased from the American Type Culture Collection (ATCC, Manassas, VA) or were donated by other laboratories (HCC193, HCC2279, HCC15) and were used as positive and negative controls. Culture conditions were as described previously. ${ }^{21} \mathrm{An}$ index array containing both the cell lines and tissue from 30 patients with a range of ALDH1 expression in twofold redundancy was assayed with each cohort array.

\section{Antibodies and Quantitative Immunofluorescence}

The BD Transduction Laboratories clone 44 mouse monoclonal antibody to ALDH1 was used (BD Biosciences, San Jose, CA). This antibody has been extensively validated in 
previous work by our research group. ${ }^{14}$ Arrays were deparaffinized in xylene (soaking twice for 20 minutes) and rehydrated with ethanol (twice in 100\% ethanol 100\% for 1 minute and then in 95\%, 85\%, and 70\% ethanol for 1 minute each). Antigen retrieval was performed in a PT module (LabVision, Fremont, CA) with citrate buffer $\left(\mathrm{pH} \mathrm{6)}\right.$ ) at $97^{\circ} \mathrm{C}$ for 20 minutes. Endogenous peroxidase activity was blocked with 30-minute incubation in $2.5 \%$ hydrogen peroxide in methanol at room temperature. Nonspecific antigens were blocked with incubation in $0.3 \%$ bovine serum albumin in Tris-buffered saline/Tween for 30 minutes at room temperature. Slides were then incubated overnight with a cocktail of the ALDH1 mouse monoclonal antibody (dilution 1:1000) and a rabbit monoclonal cytokeratin antibody (dilution 1:100; Dako, Carpinteria, CA). Ideal titers were determined as an optimal combination of image appearance and a quantitative ratio of signal to background for each of the primary antibodies, after a range of titers had been tried out in a series of test arrays. Next, a cocktail of Molecular Probes Alexa Fluor 546-conjugated goat antirabbit secondary antibody (Life Technologies-Invitrogen, Carlsbad, CA) diluted 1:100 in mouse EnVision reagent (Dako) was applied to the slides for 1 hour at room temperature. Cyanine 5 (Cy5)-tyramide (PerkinElmer, Boston, MA) was used at dilution 1:50 at room temperature for 10 minutes for signal amplification. Finally, Molecular Probes Prolong Gold (Life Technologies-Invitrogen) containing DAPI was used to detect nuclei. An index array was stained alongside each cohort array to allow the standardization of the assay, along with negative (no primary antibody) and positive controls.

The AQUA technology is a method of calculating protein concentration in subcellular compartments and has been described in detail elsewhere. ${ }^{22,23}$ Briefly, monochromatic images of DAPI and of the complexes target-Cy5 and cytokeratin-Alexa Fluor 546 were captured under a microscopy-based multiplex imaging device and microarray reader (PM-2000; HistoRx, New Haven, CT). A tumor mask was created to define the tumor area after binarization of the cytokeratin signal such that every pixel is either 'on' or 'off' on the basis of a clustering algorithm. AQUA scores in the tumor mask were calculated as the ratio of the sum of the target pixels in the tumor mask divided by the area of the tumor mask. AQUA scores were normalized for the exposure time, bit depth, and lamp hours to account for diminishing intensity over time for optimal standardization and reproducibility.

\section{Statistical Analysis}

Tumor heterogeneity of ALDH1 expression and assay reproducibility were assessed with Pearson's correlation coefficient $(R)$ between the AQUA scores from redundant cores and serial cuts of the arrays. $R^{2}>0.4$ was considered acceptable tumor heterogeneity, and $R^{2}>0.9$ was considered to be acceptable assay reproducibility. Logrank test was used to assess statistical significance in Kaplan-Meier survival curves. Kruskal-Wallis test and Mann-Whitney $U$-test were used to compare continuous ALDH1 AQUA scores between patients with different demographics. All statistical analyses were performed using JMP software version 9 (SAS Institute, Cary, NC). The level of statistical significance was set at $5 \%$.

\section{Results}

\section{Threshold for ALDH1 Detection}

In NSCLC, we identified four patterns of ALDH1 expression. In one group, tumors do not express the protein in either the epithelial component or the stroma. In a second group, there is only modest stromal expression. In a third group, expression in the epithelial component is variable and ranges from limited focal expression to expression in the majority of the tumor cells. In the fourth group, ALDH1 is strongly expressed throughout the tumor area. For the present study, we focused only on ALDH1 expression in the epithelial component of the tumors. We therefore consider the first and second groups as being negative for ALDH1 and the third and fourth groups as positive. Three cases representative of the range are shown in Figure 1. Below the detection threshold, ALDH1 expression is in the stromal component only (Figure 1, A and B). Near the detection threshold, ALDH1 is expressed weakly and focally in the epithelial component (Figure 1, C and D). Well above the detection threshold, ALDH1 is uniformly
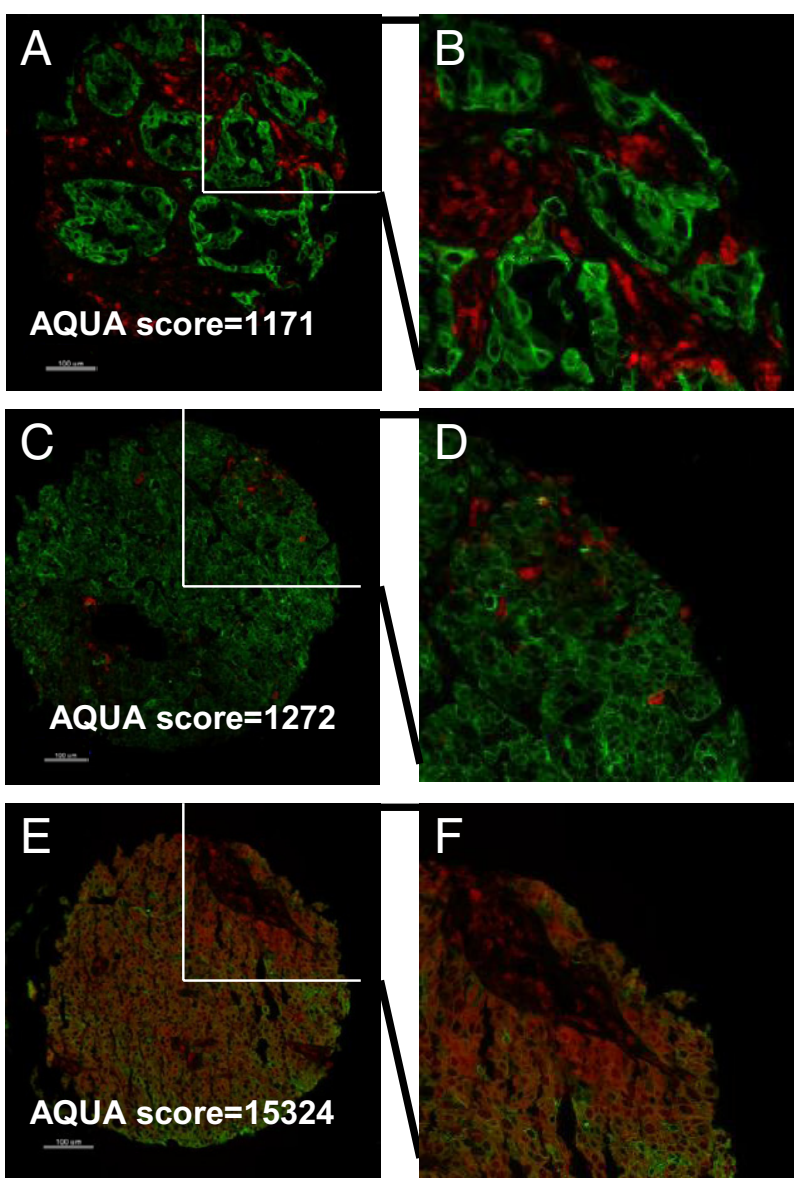

Figure 1. Three cases illustrate the range of ALDH1 expression in NSCLC. Fo each case, the entire spot is shown on the left; the boxed region corresponds to the higher magnification image on the right. A and B: ALDH1 (red) is expressed in the stromal component only, not in the epithelial component (green, defined by cytokeratin localization), and is therefore below the detection threshold. C and D: Some weak and focal expression of ALDH1 is evident in the epithelial compartment, as revealed by colocalization of cytokeratin with ALDH1. E and F: Strong and uniform expression of ALDH1 is evident in the epithelial compartment. Original magnification, $\times 200$ 
A
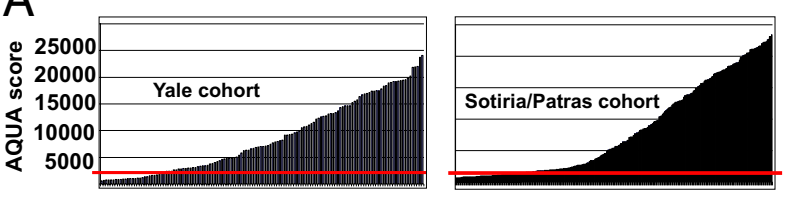

B

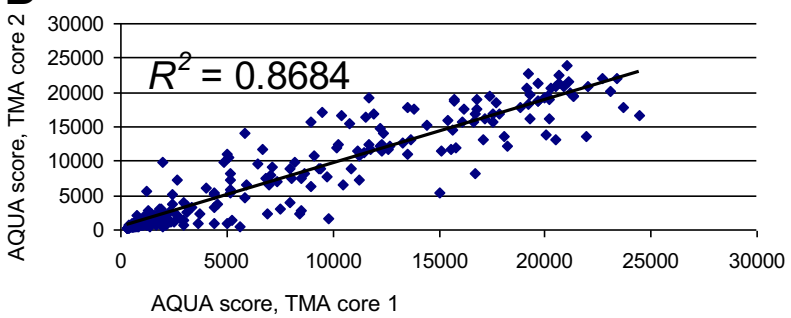

Figure 2. ALDH1 has a broad dynamic range of expression in both the Yale cohort and the Sotiria/Patras cohort. A: The dynamic range of AQUA scores for ALDH1 expression in the two cohorts. The red horizontal line indicates the signal-to-noise threshold. B: Tumor heterogeneity in the Sotiria/Patras cohort is illustrated by the correlation between AQUA scores for tissue microarray (TMA) cores that represent separate tumor areas.

expressed throughout the epithelial compartment (Figure $1, E$ and $F$ ). This last case, a high expresser, yielded an AQUA score of 15,324. The first two cases, with an AQUA score of 1171 and 1272, respectively, indicated an AQUA score of 1200 as the threshold of ALDH1 detection in our assay, and examination of many other cases near the threshold (data not shown) confirmed this cutpoint as the limit of detection for our system. Furthermore, we tested the expression of ALDH1 in a panel of cell lines and found that a threshold AQUA score of 1200 was consistent with the levels measured in cell lines. However, the broader difference between nonexpressing and expressing cell lines in our tests suggested that a better threshold could be selected using tissue samples from patient tumor cases (see Supplemental Figure S1 at http://ajp. amjpathol.org). Validation of ALDH1 patterns of expression in cell lines has been published previously. ${ }^{14}$

\section{Range of Expression and Tumor Heterogeneity}

ALDH1 had a broad dynamic range of expression in both cohorts (Figure 2A). In the Yale cohort, AQUA scores ranged from 619 to 24,090 (Table 1). Based on the AQUA score threshold, 18 cases $(13.4 \%)$ were classified as nonexpressers and 116 cases (86.6\%) as expressers. In the Sotiria/Patras cohort, AQUA scores ranged from 606 to 23,424 ; 46 cases (15.5\%) were classified as nonexpressers and 250 cases (84.5\%) as expressers. Approximately half of the expressers exhibited variable expression and the other half exhibited strong expression throughout the tumor area.

The correlation of AQUA scores between serial cuts of the index array run in independent experiments showed that the assay is highly reproducible $\left(R^{2}=0.98\right)$ (data not shown). Heterogeneity of NSCLC in ALDH1 expression assessed in the Sotiria/Patras cohort showed quite homogeneous and consistent ALDH1 expression across redundant tissue cores taken from different areas of the tumor $\left(R^{2}=0.86\right)$ (Figure $\left.2 \mathrm{~B}\right)$.

\section{Differential Expression of ALDH1 across Various Subgroups of Patients}

In both cohorts, ALDH1 was more abundant in squamous cell carcinomas than in adenocarcinomas (Figure 3). In the Yale cohort, AQUA scores ranged from 1071 to 23,738 (median, 13,232) in the squamous cell carcinoma group and from 619 to 21,989 (median, 5077) in the adenocarcinoma group ( $P=0.0059$ between the two groups) (Figure 3A). In the Sotiria/Patras cohort, scores ranged from 606 to 23,424 (median, 9139) in the squamous cell carcinoma group and from 670 to 21,133 (median, 2703) in the adenocarcinoma group $(P<0.0001$ between the two groups) (Figure 3B). Similar analysis did not reveal any differences in ALDH1 expression between patients differing in sex, stage, or age (data not shown).

\section{Prognostic Potential of ALDH1 in NSCLC}

We then tested the effect of ALDH1 expression on patient prognosis, based on the AQUA score-defined threshold of detection. Surprisingly, and contrary to cancer stem cell theories, we found that absence of ALDH1 expression identified a group of patients with worse prognosis, compared with patients who exhibited at least some minimal expression. Both cohorts showed this result when all of the patients with NSCLC were included in the analysis (Figure 4, A and B). In the Yale cohort, the group of patients with no ALDH1 expression had a median survival of 24.5 months; whereas the group with at least some ALDH1 expression had a median survival of 56.8 months $(P=0.0035)$ (Figure 4A). In the Sotiria/Patras cohort, the median survival was 23 months for the nonexpressers and 36 months for expressers $(P=0.0238$ ) (Figure 4B). When the population was divided into nonexpressers (ALDH1 negative), ALDH1 low expressers, and ALDH1 high expressers using the threshold of detection and the median score of the ALDH1-positive group as cutpoints, survival was progressively better with increasing level of expression ( $P=0.0074$ in the Yale cohort; $P=0.0019$ in the Sotiria/Patras cohort) (see Supplemental Figure S2, A and B, at http://ajp.amjpathol.org).

ALDH1 expression was present in all histology subtypes. When outcome was examined within histological classes, we found that among patients with adenocarcinoma in the
A

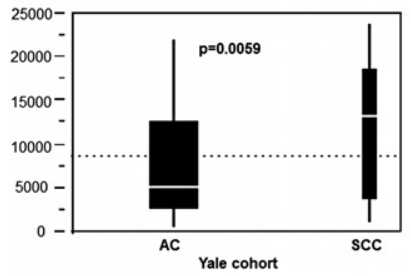

B

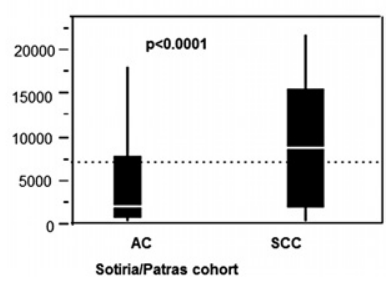

Figure 3. Differential expression of ALDH1 in patients with different histotypes. Patients with squamous cell carcinoma (SCC) are higher expressers of ALDH1 than patients with adenocarcinoma (AC) in both the Yale cohort (A) and the Sotiria/Patras cohort (B). The white horizontal lines in the boxplots represent the medians of each distribution. Comparisons were performed with the Mann-Whitney $U$-test. 
A
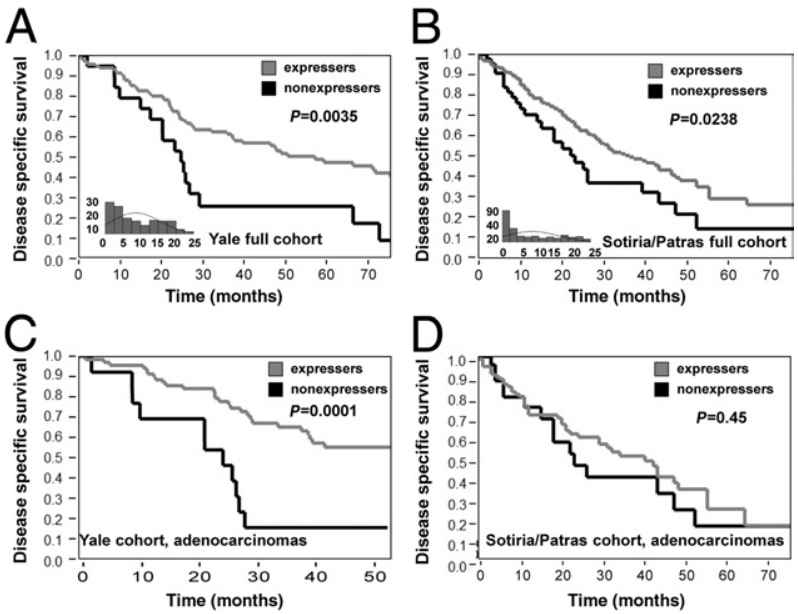

D

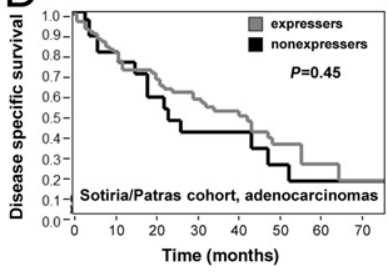

Figure 4. Absence of detectable ALDH1 defines a subgroup of patients with worse prognosis in both the Yale cohort (A) and the Sotiria/Patras cohort (B) Similar results are seen in the adenocarcinoma subset from the Yale cohort (C) and the Sotiria/Patras cohort (D). Expressers are defined by AQUA score above the signal-to-noise threshold as defined in Figure 2. Insets in $\mathbf{A}$ and $\mathbf{B}$ are frequency distributions of the ALDH1 AQUA scores. The $x$ axis is the AQUA score (divided by 1000) and the $y$ axis is the number of cases.

Yale cohort the absence of ALDH1 expression was associated with a median survival of 23 months, compared with 58.9 months for adenocarcinoma patients positive for ALDH1 ( $P=0.0001$ ) (Figure $4 \mathrm{C})$. Among patients with adenocarcinoma in the Sotiria/Patras cohort, groups with negative and positive ALDH1 status had median survival times of 23 and 40 months, respectively; this difference was not significant $(P=0.45)$ (Figure $4 \mathrm{D})$. When analysis was restricted to patients with squamous cell carcinoma, we found a trend for worse survival at the absence of ALDH1 expression in the Sotiria/Patras cohort (data not shown). Analysis was not feasible for patients with squamous cell carcinoma in the Yale cohort, because of the low number of ALDH1-negative patients in this subgroup.
Cox univariate and multivariate analyses were performed to quantify the effects of different clinicopathologic factors on survival. Age at diagnosis, sex, histotype, stage, and ALDH1 expression (after binarization into expresser and nonexpresser groups) were included in the univariate and multivariate models. In the univariate model, only stage and ALDH1 expression had a significant effect in survival (Table 2). The hazard ratio for negative status of ALDH1 in the Yale cohort was $2.24(P=0.0087)$; in the Sotiria/Patras cohort, it was $1.62(P=0.036)$ (Table 2). In the multivariate model, nonexpression of ALDH1 affected survival negatively with a hazard ratio of 3.2 in the Yale cohort $(P=0.0008)$ (Table 3$)$ and 1.51 in the Sotiria/Patras cohort $(P=0.08)$ (Table 3$)$. ALDH1 expression significantly and negatively affects survival as a continuous variable in the Cox model in both cohorts $(P=0.041$ for Yale cohort and $P=0.003$ for the Sotiria/Patras cohort).

\section{Discussion}

Expression of ALDH1 has been associated with the cancer stem cell phenotype. Ginestier et $\mathrm{al}^{11}$ found that as few as 20 cells that are ALDH1 positive and also have the CD44+/CD24-/lin ${ }^{-}$phenotype are able to form tumors when implanted in the fat pad of nude mice; in contrast, when cells are not selected for this particular phenotype, as many as 500 ALDH1-positive cells are needed. They also showed that the presence of these putative cancer stem cells in breast cancer tissue, identified using ALDH1, was associated with worse outcome. Tanei et $\mathrm{al}^{15}$ found that ALDH1-expressing breast cancers were shown to be more likely negative for ER and PR expression, but positive for HER2 and Ki-67, correlating with more aggressive breast cancer subtypes. Using $\mathrm{IHC}$ to look for cells positive for both ALDH1 and CD44 in 108 breast cancer patients treated with neoadjuvant chemo-

Table 2. Univariate Analysis

\begin{tabular}{|c|c|c|c|c|}
\hline \multirow[b]{2}{*}{ Variable } & \multicolumn{2}{|c|}{ Yale cohort } & \multicolumn{2}{|c|}{ Sotiria/Patras cohort } \\
\hline & $\mathrm{HR}(95 \% \mathrm{CI})$ & $P$ value & $\mathrm{HR}(95 \% \mathrm{Cl})$ & $P$ value \\
\hline \multicolumn{5}{|c|}{ Age at diagnosis (years) } \\
\hline$\leq 65$ & $0.82(0.56-1.2)$ & 0.32 & $0.87(0.64-1.2)$ & 0.4 \\
\hline$>65$ & 1 & & 1 & \\
\hline \multicolumn{5}{|l|}{ Sex } \\
\hline Male & $1.18(0.8-1.73)$ & 0.39 & $0.97(0.62-1.6)$ & 0.87 \\
\hline Female & 1 & & 1 & \\
\hline \multicolumn{5}{|l|}{ Histotype } \\
\hline$A C$ & $1.06(0.65-1.78)$ & 0.8 & $1.15(0.82-1.6)$ & 0.39 \\
\hline LCC & $0.7(0.26-1.63)$ & 0.43 & $2.38(0.13-10.87)$ & 0.44 \\
\hline SCC & 1 & & 1 & \\
\hline Other/mixed & $1.51(0.84-2.7)$ & 0.15 & $1.21(0.69-2.01)$ & 0.49 \\
\hline \multicolumn{5}{|l|}{ Stage } \\
\hline IA & $0.15(0.08-0.31)$ & $<0.0001$ & $0.19(0.07-0.42)$ & $<0.0001$ \\
\hline IB & $0.28(0.1-0.59)$ & $<0.001$ & $0.29(0.17-0.5)^{\prime}$ & $<0.0001$ \\
\hline II & $0.27(0.13-0.58)$ & 0.001 & $0.45(0.28-0.75)$ & 0.0024 \\
\hline III & $0.84(0.46-1.5)$ & 0.58 & $0.7(0.45-1.13)$ & 0.14 \\
\hline IV & 1 & & 1 & \\
\hline \multicolumn{5}{|l|}{ ALDH1 expression } \\
\hline No & $2.24(1.24-3.82)$ & 0.0087 & $1.62(1.03-2.45)$ & 0.036 \\
\hline Yes & 1 & & 1 & \\
\hline
\end{tabular}

AC, adenocarcinoma; $\mathrm{Cl}$, confidence interval; HR, hazards ratio; LCC, large cell carcinoma; SCC, squamous cell carcinoma. 
Table 3. Multivariate Analysis

\begin{tabular}{|c|c|c|c|c|}
\hline \multirow[b]{2}{*}{ Variable } & \multicolumn{2}{|c|}{ Yale cohort $(n=131)$} & \multicolumn{2}{|c|}{ Sotiria/Patras cohort $(n=267)$} \\
\hline & $\mathrm{HR}(95 \% \mathrm{Cl})$ & $P$ value & $\mathrm{HR}(95 \% \mathrm{Cl})$ & $P$ value \\
\hline \multicolumn{5}{|c|}{ Age at diagnosis (years) } \\
\hline $\begin{array}{l}\leq 65 \\
>65\end{array}$ & $0.75(0.46-1.22)$ & 0.24 & $\begin{array}{c}0.91(0.64-1.31) \\
1\end{array}$ & 0.64 \\
\hline \multicolumn{5}{|l|}{ Sex } \\
\hline Male & $0.37(0.59-1.58)$ & 0.9 & $0.90(0.55-1.58)$ & 0.72 \\
\hline Female & 1 & & 1 & \\
\hline \multicolumn{5}{|l|}{ Histotype } \\
\hline$A C$ & $0.8(0.43-1.55)$ & 0.5 & $0.93(0.63-1.35)$ & 0.71 \\
\hline LCC & $0.98(0.29-2.76)$ & 0.98 & $0.0003(0-21.65)$ & 0.66 \\
\hline SCC & 1 & & 1 & \\
\hline Other/mixed & $1.28(0.56-2.84)$ & 0.54 & $1.21(0.56-2.35)$ & 0.59 \\
\hline \multicolumn{5}{|l|}{ Stage } \\
\hline IA & $0.25(0.1-0.65)$ & 0.0055 & $0.21(0.08-0.51)$ & 0.0003 \\
\hline IB & $0.3(0.11-0.84)$ & 0.0023 & $0.3(0.16-0.56)$ & 0.0002 \\
\hline II & $0.19(0.06-0.58)$ & 0.004 & $0.46(0.26-0.83)$ & 0.01 \\
\hline III & $1.69(0.77-4.05)$ & 0.17 & $0.82(0.49-1.42)$ & 0.47 \\
\hline IV & 1 & & 1 & \\
\hline \multicolumn{5}{|l|}{ ALDH1 expression } \\
\hline No & $3.2(1.67-6.05)$ & 0.0008 & $1.51(0.94-2.34)$ & 0.08 \\
\hline Yes & 1 & & 1 & \\
\hline
\end{tabular}

$\mathrm{AC}$, adenocarcinoma; $\mathrm{Cl}$, confidence interval; HR, hazards ratio; LCC, large cell carcinoma; SCC, squamous cell carcinoma.

therapy, they found that residual tumors showed an increase in epithelial ALDH1 expression. They suggested that the putative cancer stem cells are capable of escaping chemotherapy and potentially driving tumor progression. ${ }^{15}$ In previous work from our research group, cells expressing both ALDH1 and CD44 were a marker for poor outcome in breast cancer. ${ }^{14}$

In other organ systems, the ALDH1 marker may not have the same implications. It has been reported that ALDH1 is a marker of better differentiation in liver cancer cells in vitro. ${ }^{24}$ Distinctive patterns in ALDH1 expression in normal tissues and correlations with their corresponding tumors have been reported, ${ }^{25}$ as well as loss of ALDH1 in ovarian cancer compared with benign ovarian tumors and normal ovaries. ${ }^{26}$ Taken together, these studies imply that ALDH1 is more likely to be present in a cancer if it is present in the corresponding normal tissue and that ALDH1 loss may be an additional step of carcinogenesis. Furthermore, in a study using the same antibody as in the present study, ALDH1 expression was shown to predict favorable prognosis in ovarian cancer. ${ }^{27}$

Because a number of different antibodies have been used for the detection of ALDH1 with IHC in human cancers and in normal tissues, ${ }^{25,28-30}$ the choice of antibody could potentially explain discrepant findings in prognostic studies. In the present study, we used clone 44 for ALDH1 detection; this antibody has been validated with Western blot for its specificity. ${ }^{14}$ In addition, we observed that the signal for ALDH1 is specific and cytoplasmic, as expected, but that it varies within a broad dynamic range. Also, in lung cancer, in contrast to breast cancer, the expression pattern is homogeneous in different areas taken from the same tumor, further validating the efficiency of our assay to adequately detect ALDH1. Deng et $\mathrm{al}^{25}$ used the same clone to detect ALDH1 and found similar patterns of ALDH1 expression in NSCLC. Although neither their study nor ours comprehensively ad- dressed the issue of tumor heterogeneity, future work could begin to address this issue by quantitatively comparing expression across tissue sections.

The literature includes discrepant findings with respect to the prognostic value of ALDH1 in lung cancer. Jiang et $\mathrm{al}^{28}$ showed that higher ALDH1 expression is associated with worse prognosis in patients with early-stage NSCLC. Similarly, Sullivan et $\mathrm{al}^{29}$ showed that ALDH1 expression had a negative effect on survival in their cohort, although it was not independent in the proportional hazards model. In both studies, the antibodies used to detect ALDH1 differed from that in the present study, and both studies used subjective determinations of expression. In both studies, cases were classified as positive or negative on the basis of a semiquantitative rule that uses the product of the percentage of cells positive and the intensity of staining, after pathology review. In the study by Jiang et $\mathrm{al}^{28}$ an arbitrary cutpoint was chosen, and approximately a third of the cases were considered to be positive, even though a number of cases with focal or weak expression were included in the positive group. (In the present study, the majority of the cases were considered to be positive on the basis of a reproducible cutpoint that represents the threshold of detection in our assay.) On the other hand, Sullivan et $\mathrm{al}^{29}$ used a statistical cutpoint (median) to divide their population into ALDH1 high and low expressers. Although both Jiang et a $\mathrm{al}^{28}$ and Sullivan et al ${ }^{29}$ concluded that higher ALDH1 expression predicts worse prognosis in NSCLC, a universal cutpoint was not determined, because the two studies were not equivalent in their methods for selection of cutpoint and neither study assessed reproducibility.

The present study has a number of limitations. First, because the group of ALDH1-negative patients comprised only $15 \%$ of the total population, the low total number of patients when grouped by histotype was insufficient for definitive analysis. Second, treatment data 
were not available in detail on either cohort, and therefore could not be included in the proportional hazards Cox model. The absence of enforced uniform treatment, as would be present in a clinical trial, weakens prognostic conclusions. Nonetheless, the fact that we see stagespecific prognostic value increases the strength of the conclusion, because treatments are more uniform within defined stages. Finally, it is difficult to explain the ALDH1 prognostic effect seen in the present study and to link it with the cancer stem cell theory.

Both Ginestier et $\mathrm{al}^{11}$ and Liang and Shi ${ }^{31}$ used expression of ALDH1 as a surrogate for identification of cancer stem cells. Although we have not performed any experiments in lung cancer cell cultures or animal models to explore cancer stem cells properties, it is possible that ALDH1 is not sufficient as a single marker to indicate cancer stem cells in all organ systems. Our finding that ALDH1 expression predicts favorable prognosis in patients with NSCLC may be a function of better differentiation in these tumors. Further studies are needed to unravel the function of ALDH1 in NSCLC and to evaluate its potential value as a prognostic marker in the routine clinical setting.

\section{References}

1. Jemal A, Bray F, Center MM, Ferlay J, Ward E, Forman D: Global cancer statistics [Erratum appeared in CA Cancer J Clin 2011, 61: 134]. CA Cancer J Clin 2011, 61:69-90

2. Kawaguchi T, Takada M, Kubo A, Matsumura A, Fukai S, Tamura A, Saito R, Kawahara M, Maruyama Y: Gender, histology, and time of diagnosis are important factors for prognosis: analysis of 1499 neversmokers with advanced non-small cell lung cancer in Japan. J Thorac Oncol 2010, 5:1011-1017

3. Ou SH, Zell JA, Ziogas A, Anton-Culver H: Prognostic factors for survival of stage I nonsmall cell lung cancer patients: a populationbased analysis of 19,702 stage I patients in the California Cancer Registry from 1989 to 2003. Cancer 2007, 110:1532-1541

4. Sakurai H, Asamura H, Goya T, Eguchi K, Nakanishi Y, Sawabata N, Okumura M, Miyaoka E, Fujii Y; Japanese Joint Committee for Lung Cancer Registration: Survival differences by gender for resected non-small cell lung cancer: a retrospective analysis of 12,509 cases in a Japanese Lung Cancer Registry study. J Thorac Oncol 2010, 5:1594-1601

5. Sculier JP, Chansky K, Crowley JJ, Van Meerbeeck J, Goldstraw P. International Staging Committee and Participating Institutions: The impact of additional prognostic factors on survival and their relationship with the anatomical extent of disease expressed by the 6th edition of the TNM Classification of Malignant Tumors and the proposals for the 7th Edition. J Thorac Oncol 2008, 3:457-466

6. Yano T, Miura N, Takenaka T, Haro A, Okazaki H, Ohba T, Kouso H, Kometani T, Shoji F, Maehara Y: Never-smoking nonsmall cell lung cancer as a separate entity: clinicopathologic features and survival. Cancer 2008, 113:1012-1018

7. Vasiliou V, Nebert DW: Analysis and update of the human aldehyde dehydrogenase (ALDH) gene family. Hum Genomics 2005, 2:138-143

8. Yoshida A, Rzhetsky A, Hsu LC, Chang C: Human aldehyde dehydrogenase gene family. Eur J Biochem 1998, 251:549-557

9. Hess DA, Meyerrose TE, Wirthlin L, Craft TP, Herrbrich PE, Creer MH, Nolta JA: Functional characterization of highly purified human hematopoietic repopulating cells isolated according to aldehyde dehydrogenase activity. Blood 2004, 104:1648-1655

10. Douville J, Beaulieu R, Balicki D: ALDH1 as a functional marker of cancer stem and progenitor cells. Stem Cells Dev 2009, 18:17-25

11. Ginestier C, Hur MH, Charafe-Jauffret E, Monville F, Dutcher J, Brown M, Jacquemier J, Viens P, Kleer CG, Liu S, Schott A, Hayes D, Birnbaum D, Wicha MS, Dontu G: ALDH1 is a marker of normal and malignant human mammary stem cells and a predictor of poor clinical outcome. Cell Stem Cell 2007, 1:555-567
12. Charafe-Jauffret E, Ginestier C, lovino F, Tarpin C, Diebel M, Estern B. Houvenaeghel G, Extra JM, Bertucci $F$, Jacquemier J, Xerri L, Dontu G, Stassi G, Xiao Y, Barsky SH, Birnbaum D, Viens P, Wicha MS: Aldehyde dehydrogenase 1-positive cancer stem cells mediate metastasis and poor clinical outcome in inflammatory breast cancer. Clin Cancer Res 2010, 16:45-55

13. Neumeister V, Rimm D: Is ALDH1 a good method for definition of breast cancer stem cells? Breast Cancer Res Treat 2010, 123:109-111

14. Neumeister V, Agarwal S, Bordeaux J, Camp RL, Rimm DL: In situ identification of putative cancer stem cells by multiplexing ALDH1, CD44, and cytokeratin identifies breast cancer patients with poor prognosis. Am J Pathol 2010, 176:2131-2138

15. Tanei T, Morimoto K, Shimazu K, Kim SJ, Tanji Y, Taguchi T, Tamaki $Y$, Noguchi S: Association of breast cancer stem cells identified by aldehyde dehydrogenase 1 expression with resistance to sequential Paclitaxel and epirubicin-based chemotherapy for breast cancers. Clin Cancer Res 2009, 15:4234-4241

16. Marcato $P$, Dean CA, Pan D, Araslanova R, Gillis M, Joshi M, Helyer L, Pan L, Leidal A, Gujar S, Giacomantonio CA, Lee PW: Aldehyde dehydrogenase activity of breast cancer stem cells is primarily due to isoform ALDH1A3 and its expression is predictive of metastasis. Stem Cells 2011, 29:32-45

17. Alison MR, Guppy NJ, Lim SM, Nicholson LJ: Finding cancer stem cells: are aldehyde dehydrogenases fit for purpose? J Pathol 2010, 222:335-344

18. Anagnostou VK, Welsh AW, Giltnane JM, Siddiqui S, Liceaga C, Gustavson M, Syrigos KN, Reiter JL, Rimm DL: Analytic variability in immunohistochemistry biomarker studies. Cancer Epidemiol Biomarkers Prev 2010, 19:982-991

19. Brambilla E, Travis WD, Colby TV, Corrin B, Shimosato Y: The new World Health Organization classification of lung tumours. Eur Respir J 2001, 18:1059-1068

20. Edge SB, Compton CC: The American Joint Committee on Cancer: the 7th edition of the AJCC cancer staging manual and the future of TNM. Ann Surg Oncol 2010, 17:1471-1474

21. McCabe A, Dolled-Filhart M, Camp RL, Rimm DL: Automated quantitative analysis (AQUA) of in situ protein expression, antibody concentration, and prognosis. J Natl Cancer Inst 2005, 97:1808-1815

22. Camp RL, Chung GG, Rimm DL: Automated subcellular localization and quantification of protein expression in tissue microarrays. Nat Med 2002, 8:1323-1327

23. Dolled-Filhart M, Gustavson M, Camp RL, Rimm DL, Tonkinson JL, Christiansen J: Automated analysis of tissue microarrays. Methods Mol Biol 2010, 664:151-162

24. Chen X, Lingala S, Khoobyari S, Nolta J, Zern MA, Wu J: Epithelial mesenchymal transition and hedgehog signaling activation are associated with chemoresistance and invasion of hepatoma subpopulations. J Hepatol 2011, 55:838-845

25. Deng S, Yang X, Lassus H, Liang S, Kaur S, Ye Q, Li C, Wang LP, Roby KF, Orsulic S, Connolly DC, Zhang Y, Montone K, Bützow R, Coukos G, Zhang L: Distinct expression levels and patterns of stem cell marker, aldehyde dehydrogenase isoform 1 (ALDH1), in human epithelial cancers. PLoS One 2010, 5:e10277

26. Penumatsa K, Edassery SL, Barua A, Bradaric MJ, Luborsky JL: Differential expression of aldehyde dehydrogenase 1a1 (ALDH1) in normal ovary and serous ovarian tumors. J Ovarian Res 2010, 3:28

27. Chang B, Liu G, Xue F, Rosen DG, Xiao L, Wang X, Liu J: ALDH1 expression correlates with favorable prognosis in ovarian cancers. Mod Pathol 2009, 22:817-823

28. Jiang F, Qiu Q, Khanna A, Todd NW, Deepak J, Xing L, Wang H, Liu Z, Su Y, Stass SA, Katz RL: Aldehyde dehydrogenase 1 is a tumor stem cell-associated marker in lung cancer. Mol Cancer Res 2009, 7:330-338

29. Sullivan JP, Spinola M, Dodge M, Raso MG, Behrens C, Gao B, Schuster K, Shao C, Larsen JE, Sullivan LA, Honorio S, Xie Y, Scaglioni PP, DiMaio JM, Gazdar AF, Shay JW, Wistuba II, Minna JD: Aldehyde dehydrogenase activity selects for lung adenocarcinoma stem cells dependent on notch signaling. Cancer Res 2010, 70:99379948

30. Patel M, Lu L, Zander DS, Sreerama L, Coco D, Moreb JS: ALDH1A1 and ALDH3A1 expression in lung cancers: correlation with histologic type and potential precursors. Lung Cancer 2008, 59:340-349

31. Liang D, Shi Y: Aldehyde dehydrogenase- 1 is a specific marker for stem cells in human lung adenocarcinoma. Med Oncol 2012, 29:633-639 